\title{
NOVEL USE OF RESONANT TUNNELING STRUCTURES FOR OPTICAL AND IR MODULATORS
}

\author{
I. Mehdi, G. I. Haddad and R. K. Mains \\ Center for High Frequency Microelectronics \\ Department of Electrical Engineering and Computer Science \\ The University of Michigan \\ Ann Arbor, Michigan 48109
}

(Received 8 August 1988)

\begin{abstract}
The basic concepts and some preliminary calculations are presented showing the feasibility of high speed optical and infrared modulators based on resonant tunneling structures. A modulator based on a conventional resonant tunneling structure can be operated in the infrared region by using the intersubband transitions and in the optical region by using the valence band to conduction band transitions in the quantum well of the double barrier structure. A novel resonant tunneling structure is proposed as a modulator in which the quantum well conduction band at zero bias is below the collector layer conduction band. This deep well resonant tunneling structure can allow intersubband transitions with higher energy than its conventional counterpart and with a careful design may even result in population inversion and gain. The band-to-band transitions are also possible in the deep well resonant tunneling modulator. Due to the inherent negative resistance of the device which persists to ultrahigh frequencies, it is possible to operate the device as a self-oscillating modulator.
\end{abstract}

\section{Introduction}

The ultrafast phenomenon of resonant tunneling has been demonstrated in a host of electronic devices. ${ }^{1-4}$ Preliminary investigations show that for high frequency applications the device will be limited by external parameters rather than any intrinsic limitations. Many of the investigations have been on the use of the structures as electronic devices. Due to their fast response time and device physics it is possible to utilize the resonant tunneling structures as optical components. As optical components their ultrafast speed could be put to a better use since probably the extrinsic time constants of optical systems will be shorter compared to electronic circuits. Another area where resonant tunneling structures could become very useful would be at the interface of electronic and optical systems. Due to the unique device physics of the resonant tunneling structures it is possible to use the device simultaneously as an oscillator and optical modulator resulting in a self-oscillating modulator.

In this investigation we report some preliminary calculations showing the feasibility of using resonant tunneling structures (RTS)as optical components. A device. concept is presented utilizing the RTS as an optical modulator. Because of the many options that are available in designing RTS, the optical modulator can work in a number of different modes making use of band-to-band transitions or intersubband transitions. Discussion of each mode and some preliminary calculations will be presented.

The basic resonant tunneling structure is a thin quantum well sandwiched between thin barriers. Due to the barrier confinement quasi-bound states are created in the well. The position in energy space of these quasibound states depends on the device dimensions as well as the material system that is utilized. For the conventional case at zero bias the ground quasi-bound state is above the Fermi level in the contact. At a bias point where the Fermi level lines up with the quasi bound state a peak in the current is obtained. As the device bias is further increased the current level decreases due to the nonalignment of the emitter Fermi level with the quasibound state.

\section{RTS as an Optical Modulator}

The modulators discussed in this investigation can generally be divided into two groups depending on the 
the nature of the optical transition. The first group involves the transitions from the valence band to the conduction band of the quantum well. These will be termed the interband resonant tunneling modulators. The other case involves transitions within the conduction band of the quantum well. These will be termed the intersubband resonant tunneling modulators. Two different device structures will be discussed in each group.

\section{2a. The Model}

The basic physical phenomenon involved in the operation of the resonant tunneling modulators is the ability to fill and empty the quasi-bound states with electrons. To solve the transport problem in the conventional resonant tunneling structure the model of Tsu and Esaki ${ }^{5}$ was used. The approach involves the solution of the Schrodinger equation in each region of the device. A constant effective mass is used and scattering is neglected. No attempt is made to include self consistency in this model. The tunneling transmission coefficient is determined by the transfer matrix method. A $2 \times 2$ matrix at each interface is formed by matching the continuity of the wave functions and their derivatives at each boundary point. Successive multiplication of these matrices then couples the incident wave to the outgoing wave. A plot of the transmission coefficient versus incident energy shows distinctive peaks in the transmission coefficient corresponding to the quasi-bound states in the quantum well.

Once the overall transmission coefficient of the device has been found, the stcady-statc wave functions can be determined at each incident energy over the full range of interest. The electronic charge density can be determined using

$$
\rho(z)=q \sum_{E_{i}}\left|\psi\left(E_{i}, z\right)\right|^{2} \frac{m^{*} k T}{\pi \hbar^{2}} \log \left(1+e^{\left(E_{i} E_{f}\right) / k T}\right) \Delta E
$$

where $E_{i}$ is the incident energy, $k$ is the Boltzman constant, $\mathrm{T}$ is temperature and $\mathrm{m}^{*}$ is the effective mass. $E_{f}$ is the Fermi energy corresponding to the doping in the emitter or collector layer depending on the direction of the incident electrons. Electrons incident from both directions were accounted for in our calculation. The one dimensional distribution function is obtained assuming that the $x-y$ dimensions are essentially decoupled from the $z$ direction and can then be considered to be in a quasi-equilibrium state; the transverse dimensions are then integrated over the equilibrium Fermi distribution.

The terminal current density is calculated at each applied bias using ${ }^{5}$

$J=\frac{e m^{*} k T}{2 \pi^{2} \hbar^{3}} \int_{0}^{\infty} \tau \ln \left(\frac{1+\exp \left(\left(E_{F}-E_{i}\right) / k T\right)}{1+\exp \left(\left(E_{F}-E_{i}-e V_{a p p}\right) / k T\right)}\right)$ (a)

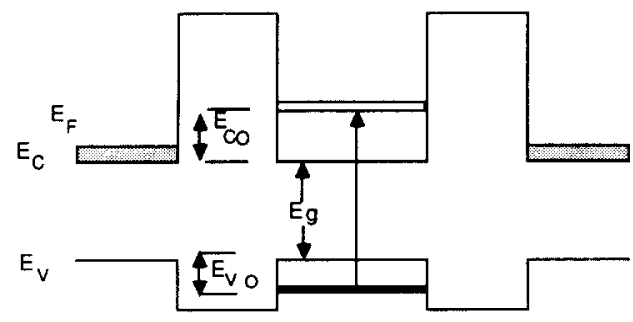

(b)

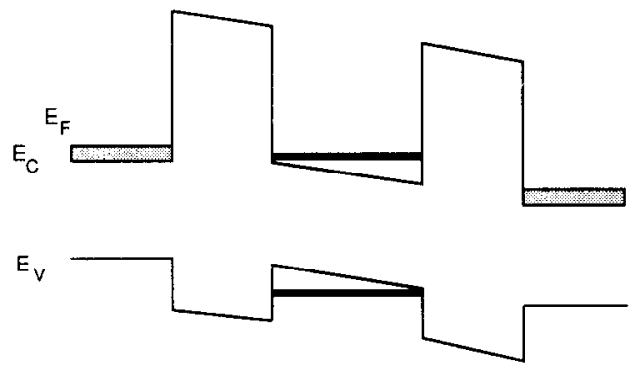

Fig. 1. A conventional double barrier single qunatum well resonant tunneling structure(RTS) (a) at zero bias, (b) applied bias.

where $V_{a p p}$ is the applied voltage across the entire device and $\tau$ is the transmission coefficient. All calculations are carried out at room temperature.

\section{2b. The RTS Modulator with Interband Transitions}

In this mode of operation transitions from the valence band to the conduction band are utilized. Consider the conventional resonant tunneling structure as presented in Figure 1(a). A typical I(V) curve for this structure along with the carrier concentration of the ground state as a function of applied bias is shown in Figure 2. Such a structure can be obtained from a GaAs well and AlAs barriers or from a InGaAs well and InAlAs barriers. The operation of the modulator will be as follows. At zero bias the structure will be as presented in Figure 1(a). The quasi bound state in the quantum well will be high enough in the well that due to the assumed Fermi distribution of the carriers in the emitter and collector layer the carrier concentration in the well will be small compared to the carrier concentration in the emitter and collector layers. At zero bias the current through the structure will be zero since the current from left to right will cancel the current from right to left. The valence band, however, at zero bias will be full of electrons. Due to the valence band discontinuities there will be quasibound states in the valence band but they will be fully occupied by electrons. Now if an optical signal is applied with the energy corresponding to 


$$
E_{\text {incident }}=E_{g}+E_{c_{o}}+E_{v_{o}}
$$

where $E_{q}$ is the band gap(of the well material) and $E_{c_{o}}$ and $E_{v_{0}}$ are the energy levels of the first conduction band quasi-bound state and the first valence band quasibound state respectively (all with respect to the middle of the quantum well) then transitions can take place from the valence band to the conduction band. The optical signal will excite the electrons from the valence band into the conduction band quasi-bound state. Thus one can imagine that light with a particular energy, $E_{\text {incident }}$ will be absorbed by this structure.

If the structure is biased at the point where the carrier concentration in the well is at a peak value, the band diagram represented in Figure 1(b) will be obtained. The bias corresponds to the bias needed to obtain peak current as shown in Figure 2. At this bias point the valence band is still full of electrons but now the quasi bound state in the quantum well is aligned to the Fermi level in the emitter layer and thus the quasi bound state is not empty. In this case the optical signal will not be absorbed, or the absorption will decrease significantly because the electrons from the valence band will not find a corresponding empty state in the conduction band quasi-bound state. This will be the nonabsorbing state of this mode.

The most general form of the absorption coefficient $\alpha$ for a given photon energy for interband transitions is proportional to the probability $P_{i f}$ for the transition from the initial state to the final state and to the density of electrons in the initial state, $n_{i}$, and also to the density of available(empty) final states, $n_{f}$, and this process must be summed for all possible transitions between states separated by an energy difference equal to $h \nu:^{6}$

$$
\alpha(h \nu)=A \sum P_{i f} n_{i} n_{f}
$$

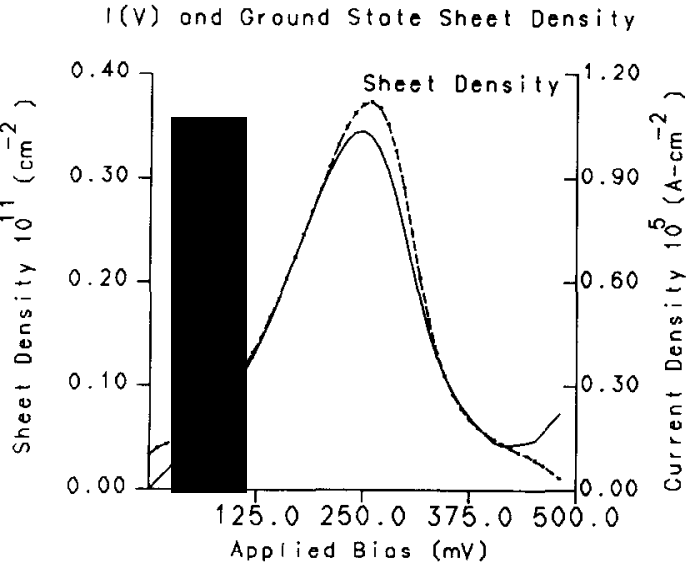

Fig. 2. I(V) curve for a resonant tunneling structure and the sheet charge density of the ground state.
Assuming that $P_{i f}$ is constant over the range of the applied bias, then $\alpha$ can be written as

$$
\alpha(h \nu)=C \sum n_{i} n_{f}
$$

where $\mathrm{C}$ is a constant and the summation is carried over all the possible transitions for a particular incident energy. For further simplicity it is assumed that all the lower(valence) band states are filled and $n_{f}$ is inversely proportional to the sheet charge density of the particular final state energy under consideration. This by no means is a rigorous calculation but it is felt that it qualitatively depicts the system. Also in our calculations the quantum confined Stark shift has been neglected.

A RTS with $20 \AA$ barriers and a $40 \AA$ well is considered. The barrier height is selected to be $500 \mathrm{meV}$. The doping in the emitter layer and the collector layer is $5.0 \times 10^{17}$. The peak in the current occurs at an applied bias of $0.25 \mathrm{~V}$. At zero bias the sheet density of the ground state is $4.25 \times 10^{9} \mathrm{~cm}^{-2}$ while at the peak bias point the sheet density increases to $3.69 \times 10^{10} \mathrm{~cm}^{-2}$. This represents about an order of magnitude increase in the carrier density and thus the absorption coefficient will decrease accordingly at the peak bias point. The absorption coefficient of this structure is shown in figure 3 as curve $A$.

A similar result can be obtained by using a deep quantum well device structure. The band diagram of such a structure is shown in Figure 4. Stuch structures

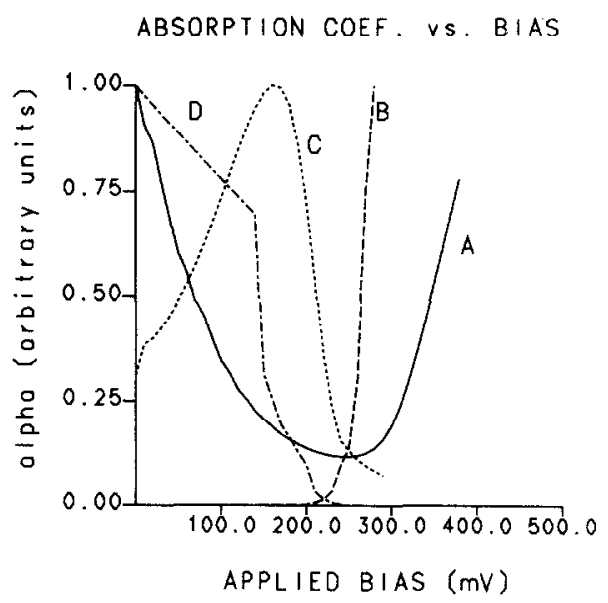

Fig. 3. Absorption coefficient in arbitrary units for the different device structures. Curve A refers to the interband transitions in the conventional RTS, curve B corresponds to the mode with interband transitions in a deep quantum well RTS, curve C and curve D pertain to the intersubband mode of operation for the conventional and deep well resonant tunneling structures respectively. 
(a)
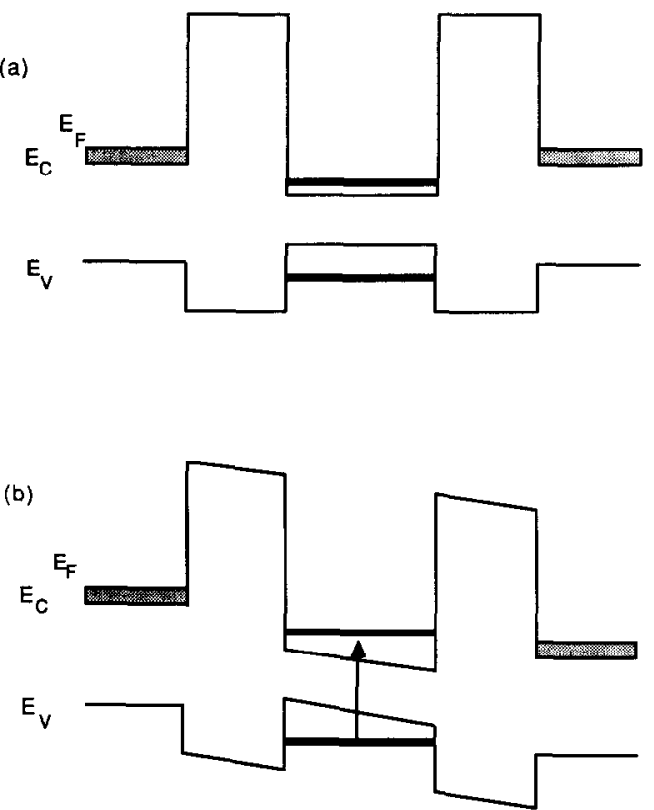

Fig. 4. Band diagram of a deep well resonant tunneling structure (a) at zero bias (b) applied bias.

have been fabricated by using a $\mathrm{In}_{0.20} \mathrm{Ga}_{0.80}$ As well and AlAs barriers on a GaAs substrate. In such a case the ground state falls below the conduction band of the contact layers. We have showed the feasibility of such devices and the existence of the bound state. ${ }^{7}$ The modulator based on such a structure will function as follows. At zero bias the ground state is below the collector and emitter conduction band and will thus be occupied by electrons. This state in fact becomes a bound state. The ground state in the valence band of course is full of electrons as before. Now when an optical signal corresponding to the energy

$$
E_{\text {optical }}=E_{g}+E_{c_{b o}}+E_{v_{o}}
$$

where $E_{c_{b o}}$ is the energy of the bound state in the deep quantum well relative to the middle of the quantum well (as shown in fig. 1a), is applied the electrons in the valence band will be excited but will not find any corresponding empty states in the conduction band. Thus the optical signal will not be absorbed. As an electric field is applied across the device the band bends and the states in the quantum well move. At a particular bias note that the Fermi level of the collector region aligns with the ground state in the well and due to the applied field the ground state will be emptied out. Once the ground state is emptied, on the application of an appropriate optical signal the electrons from the valence band can find corresponding empty energy levels and thus the light will be absorbed. Thus by applying the appropriate bias the device will absorb optical energy.

A device with $30 \AA$ barriers and a $70 \AA$ well is studied as an example. The barrier height relative to the emitter layer is about $500 \mathrm{meV}$ and the well is taken to be $153 \mathrm{meV}$ below the conduction band of the emitter. The method to calculate the bound energy state and the carrier concentration of the bound energy state has been described eleswhere. ${ }^{8}$ The doping in the collector and emitter layers is taken to be $1.0 \times 10^{18} \mathrm{~cm}^{-3}$. An effective mass of $0.042 m_{0}$ is used. This device structure can be realized by using a strained InGaAs well and AlAs barriers on GaAs substrates. As bias is applied the ground state shifts only a few meV in energy with respect to the bottom of the well but in order to find out when the bound state becomes quasi-bound, movement of the bound state quantized level with respect to the collector conduction band edge has to be studied. It is found that the difference between the bound state energy and the conduction band decreases linearly with applied bias and at a certain bias point the bound state energy lines up with the collector conduction band thus becoming a quasi-bound state. For the particular device simulated the ground state is bound as long as the applied bias is less than $150 \mathrm{meV}$. As the bias increases from $150 \mathrm{meV}$ the ground state becomes a quasi-bound state and becomes less populated. This change in the carrier concentration of the ground level will thus cause a change in $\alpha$. The sheet carrier density of the bound state as a function of applied bias is shown in figure 5 . At zero bias the state is below the conduction band and thus an extremely high shet carrier density of $2.6 \times 10^{12} \mathrm{~cm}^{-2}$ is obtained. As bias is applied the state moves closer

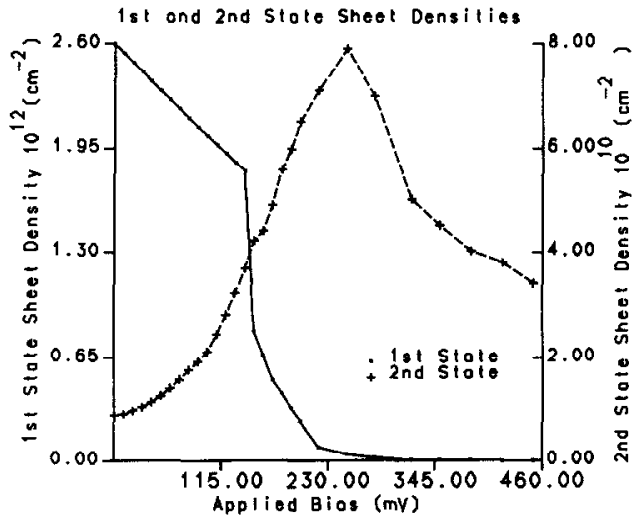

Fig. 5. Sheet carrier density of the bound state(1st state) and the second state in the deep well RTS as a function of applied bias. The well is taken to be 153 $\mathrm{meV}$ below the conduction band of the collector and emitter layer at zero bias. Effective mass of $0.067 \mathrm{~m}$ 。 was used. 
to the conduction band of the collector layer and thus the carrier concentration decreses. As the bound state becomes a quasi-bound state the charge concentration decreases to $7.2 \times 10^{11} \mathrm{~cm}^{-2}$. With increasing bias the sheet concentration of the quasi-bound state decreases. The absorption coefficient, in arbitrary units for this device structure is shown in figure 3 as curve $B$.

\section{2c. The RTS Modulator with Intersubband Transitions}

It has been documented that transitions of infrared radiation can be obtained from the conduction band of a semiconductor quantum well because of the existnece of the quantized subbands in the conduction band ${ }^{9-11}$. The modulator concept presented here utilizes this fact and combines the fast carrier transfer capability of resonant tunneling structures to control the transitions between the subbands. In this mode of operation, again, two structues can be utilized. The conduction band of a conventional resonant tunneling structure is shown in figure 6 . In this case care must be taken in designing the devices so that the first excited state can be utilized. Experimental evidence of resonant tunneling through the first excited state has been reported. ${ }^{12}$ The use of the conventional resonant tunneling diode will be as follows. At zero bias the conduction band is shown in figure 6(a). Two quasibound states are present in the quantum well. Since at zero bias the ground state is considerably above the Fermi level in the emitter region both quasi bound states will be devoid of any carriers. On applying the appropriate optical signal with energy equal to

$$
E_{\text {incident }}=E_{c_{1}}-E_{c_{0}}
$$

where $E_{c_{0}}$ and $E_{c_{1}}$ refer to the ground state and the first excited state energy levels respectively, no absorbtion will take place because the ground state will not have any electrons even though empty states will be available in the first excited state. Thus light will not be absorbed in the structure. When an appropriate bias is applied the band structure shown in Figure 6(b) is obtained. At this bias point the first peak in the current is observed due to the alignment of the ground state in the quantum well with the Fermi energy of the emitter layer. At this bias the carrier concentration in the ground state is increased while the carrier concentration in the first excited state is low. Thus, on the application of the appropriate optical signal, the electrons can be excited from the ground state to the first excited state absorbing optical energy in the process.

It is well known that the optical absorption coefficient in this case depends on the matrix element, the density of states( or population of the initial and final states), and the linewidth. ${ }^{13}$ For intersubband transitions, the matrix element $\left|\left(\psi_{2}|e z| \psi_{1}\right)\right|$ varies slowly with applied voltage and thus the absorption coefficient is (a)

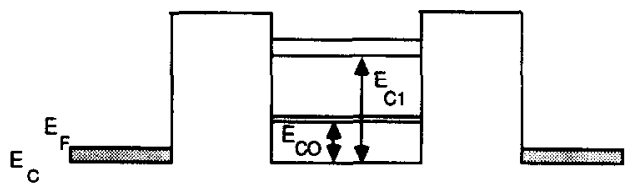

(b)

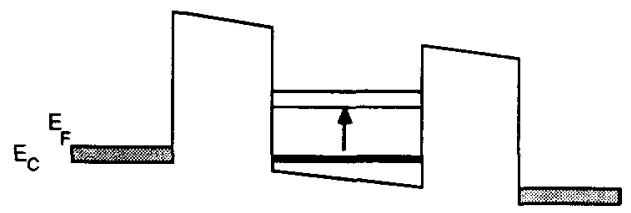

Fig. 6. Conduction band of a conventional RTS at (a) zero bias (b) applied bias.

more directly related to the other two parameters. Ahn and Chaug ${ }^{12-14}$ have derived the intersubband optical absorption eoefficient $\alpha$ for the transition between ground state and the first excited state as

$$
\alpha=\frac{\omega \mu c}{n_{\tau}} \frac{2}{V} \sum_{k_{z}}\left|M_{21}\right|^{2} \frac{\left(f_{a}-f_{b}\right) \hbar \gamma_{a b}}{\left(E_{2}-E_{1}-\hbar \omega\right)^{2}+\left(\hbar \gamma_{a b}\right)^{2}}
$$

where $M_{21}$ is the matrix element involving the envelope wavefunctions of the two states, $\mu$ is the permeability, $n_{r}$ is the refractive index, $\mathrm{c}$ is the speed of light, $\mathrm{V}$ is the volume, $k_{t}$ is the wave vector of the electron in the $x-y$ plane, $E_{1}$ and $E_{2}$ denote the quantized energy levels for the intitial and final states respectively, $a$ and $b$ are the short hand notations for the total wave vectors of the initial and final states respectivley, $\hbar \gamma_{\mathrm{ab}}$ is the relaxation rates, and $f_{s}$ and $f_{b}$ are the Fermi-Dirac distribution functions for the initial and final states respectively. It is important to realize that the above formalism is strictly not valid for resonant tunneling structures where the barriers are not infinite and thus tunneling is very significant. If one changes the summation over $k_{t}$ in the above equation into the integration over the transverse energy $E_{t}$ and assumes that $\bar{h} \gamma_{\mathrm{ab}}$ is independent of the applied voltage $e^{14,15}$ then one can analytically integrate equation 10 and $\alpha$ turns out to be proportional to the population difference in the two quantized levels. ${ }^{13}$

To find the change in the populations of the quasibound states, a calculation was carried out for a resonant tunneling structure of well width $50 \AA$ and barrier widths of $25 \AA$. The barrier height used was $350 \mathrm{meV}$ and $m^{*}=0.067 m_{0}$. The doping in the collector and emitter layers was $5.0 \times 10^{17} \mathrm{~cm}^{-3}$. Figure 7 shows the sheet carrier concentration of the ground state and the first excited state as a function of applied bias. At zero bias the ground state concentration is about $2.22 \times 10^{9} \mathrm{~cm}^{-2}$ while the carrier density in the first excited state is about $1.5 \times 10^{6} \mathrm{~cm}^{-2}$. As bias is increased the first state 


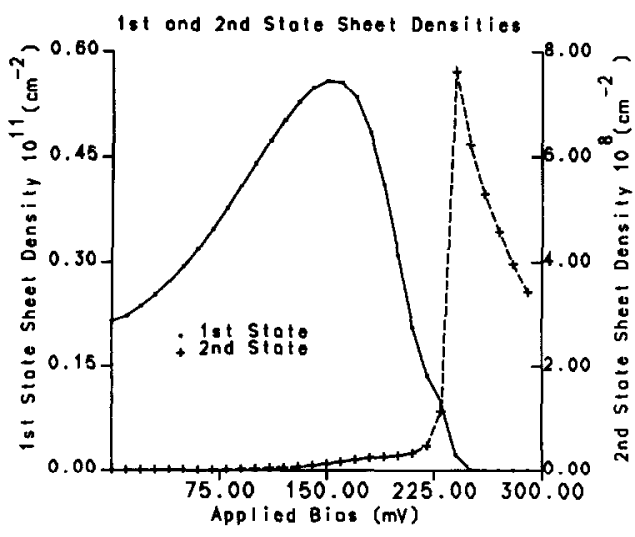

Fig. 7. Sheet carrier densities of the ground state(first state) and the second state in a conventional RTS.

reaches a peak and then the concentration of the ground layer drops. Maximum absorption would be expected at the point where the ground state achieves the maximum sheet concentration. The resultant $\alpha$ in arbitrary units is shown in figure 3 as curve $C$.

The deep well resonant tunneling structure proposed earlier for interband transitions can be used for the intersubband case. A conduction band diagram of such a structure is shown in Figure 8. Again by proper device design the device can be tailored to have the ground state below the emitter and conduction band edges. This state as explained above is now a bound state completely filled with electrons at zero bias. On the application of an appropriate optical signal of energy

$$
E_{\text {incident }}=E_{c_{1}}^{\prime}-E_{c_{b o}}^{\prime}
$$

where $E_{c_{1}}$ and $E_{c_{b_{0}}}$ represent the first excited state and the bound state energies respectively, the electrons can be excited from the bound level to the first excited state. Thus at zero bias optical energy will be absorbed. However, with application of an electric field the bound state energy moves relative to the conduction band of the collector layer as discussed and at a particular bias( 105 $\mathrm{mV}$ in this case) the bound state becomes a quasibound state and the carrier concentration of the bound state decreases substantially. From the above theory $\alpha$ will again be expected to be proportional to the population difference between the two states within the framework of our approximations. Thus modulation can be obtained since $\alpha$ changes as a function of bias. Figure 5 shows the carrier sheet concentration in the bound state and in the first excited state as a function of applied bias for this device structure. With the application of an electric field, a significant change in the sheet concentration of the bound state occurs. Consequently, a significant change in the absorption coefficient would be (a)

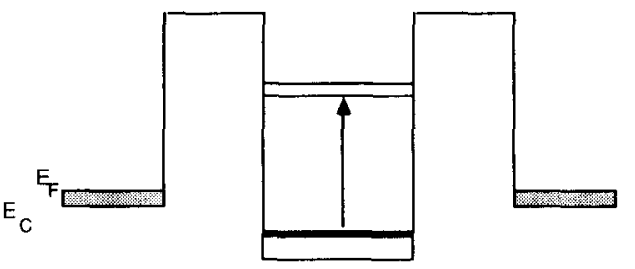

(b)

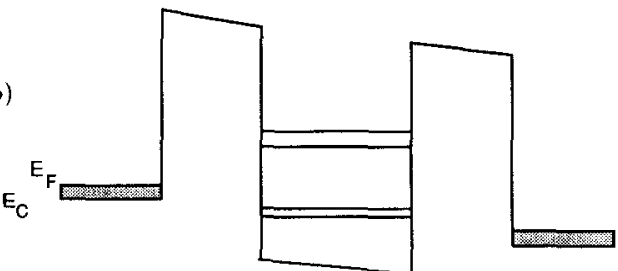

Fig. 8. Conduction band diagram of a deep quantum well RTS at (a) zero bias and (b) at some particular applied bias.

expected in such a device. The absorption coefficient of such a structure is presented in figure 3 as curve $D$.

In the device structures considered as examples no attempt was made to optimize the results. The device dimensions and other parameters used in the simulation are experimentally relizable. To optimize the performance of the stuructures a systematic study would have to be done to understand how the device dimensions and other physical parameters effect the modulation properties of the structures.

\section{Other Possible Devices}

The deep well RTS can also be used as an electromagnetic energy source rather than a modulator. In this case a suitable device can be designed that at a particular applied hias has a conduction band structure shown in figure 9. At the bias point shown the ground state will be relatively less populated because of the applied electric field, however, the first excited state will be full of carriers since it will be lined up with the Fermi level of the emitter. Thus stimulated emission could take place from the first excited state to the ground state since population inversion will be established. The barriers will have to be high enough in energy so that the device symmetry does not diminish significantly with applied bias.

Since the RTS is a negative resistance device where the negative resistance persists to ultrahigh frequencies ${ }^{\mathbf{1 2 , 8},}$, oscillators can be realized at these frequencies. It is therefore possible to obtain self-oscillting optical and infrared modulators operating at extremely high frequencies using these structures. It is also possible to tune 


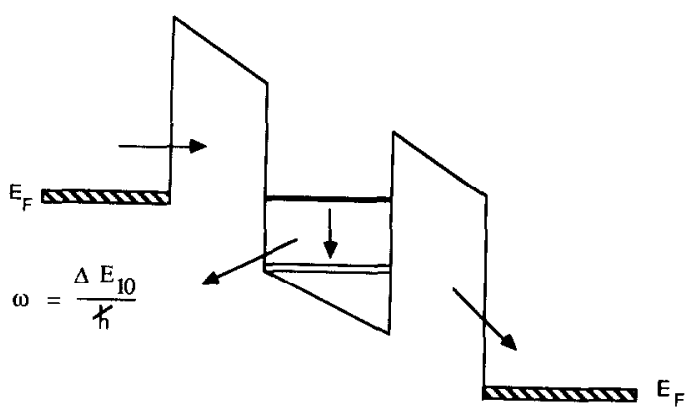

Fig. 9. Conduction Band diagram of deep quantum well RTS that could be used as a source of electromagnetic energy.

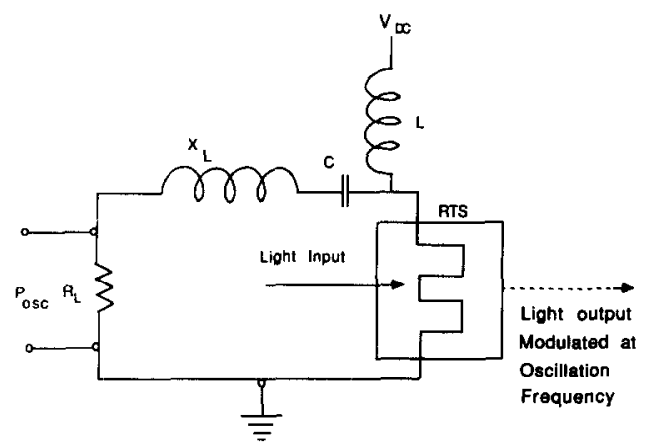

Fig. 10. Idealized circuit for a self-oscillating modulator and light tunable or modulated oscillator.

and modulate an oscillator utilizing such devices by applying optical signals. These particular applications are indicated in figure 10.

\section{Conclusion}

Preliminary calculations and device ideas for utilizing resonant tunneling structures as optical and infrared modulators have been presented. The modulators can work over a wide range of incident energy. A deep well RTS is presented as a modulator that can work better than its conventional counterpart. This is possible because of the creation of a bound state in the deep quantum well at zero bias that changes to a quasi bound state with applied bias substantially affecting the carrier concentration of the energy state. Both the interband transition mode and the intersubband transition mode of the modulators have been discussed. A proposal for an electromagnetic energy source and a self oscillating modulator using RTS has also been made.

Acknowledgments- The authors would like to thank S. Hong and Prof. Singh for many helpful technical discussions on this work. This work was supported by the US Army Research Office under the URI program, contract no. D $\Lambda$ AL03-87-K-0007.

\section{References}

[1] T. C. L. G. Sollner, E. R. Brown, and H. Q. lee, The Lincoln Laboratory Journal, 1,89 (1988).

[2] E. R. Brown, T. C. L. G. Sollner, W. D. Goodhue, and C. D. Parker, Applied Physics Letters, 50, 83,1987

[3] F. Capasso, K. Mohammad, and A. Y. Cho, IEEE Journal of Quantum Electronics, QE- 22, 1853, 1986.

[4] K. K. Choi, B. F. Levine, R. J. Malik, J. Walker, and C. G. Bethea, Physical Review B, 35,4172(1987)

[5] R. Tsu and L. Esaki, Applied Physics Letters, 22, $562,(1973)$

[6] J. I. Pankove, "Optical processes in semiconductors, ${ }^{n}$ Dover Publications, 1971.

[7] I. Mehdi and G. I. Haddad, to be published.

[8] R. K. Mains and G. I. Haddad, to appear in Journal of Applied Physics.

[9] L. C. West and E. J. Eglash, Applied Physics Letters 46, 1156, (1984)

[10] B. F. Levine, R. J. Malik, J. Walker, K. K. Choi, C. G. Bethea, D. A. Kleinman, and J. M. Vandenberg, Applied Physics Letters,50, 273, 1987

[11] M. Yu. Martisov and A. Ya. Shik, Soviet Physics Semiconductors 21, 209, (1987)

[12] M. A. Reed, Superlattices and Microstructures, 2, $65,(1986)$.

[13] D. Ahn and S. L. Chuang, Third International Conference on Superlattices, Microstructures and Microdevices, Chicago August 17, (1987).

[14] D. Ahn and S. L. Chuang, IEEE Journal of Quantum Electronics,23,2196(1987).

[15] D. Ahn and S. L. Chuang, Physical Review B, 35,4149(1987). 